\title{
Review of Modular Self-Reconfigurable Robotic Systems and Potential Industry Problems
}

\author{
${ }^{1}$ John Huria Nderitu \\ 1,2 University of Nairobi, Nairobi, Kenya. \\ ${ }^{1}$ laneeritu@uonbi.ac.ke
}

\author{
ArticleInfo \\ Journal of Machine and Computing (http://anapub.co.ke/journals/jmc/jmc.html) \\ Doi : https://doi.org/10.53759/7669/jmc202101014 \\ Received 15 March 2021; Revised form 22 April 2021; Accepted 05 June 2021; Available online 05 July 2021. \\ (C)2021 The Authors. Published by AnaPub Publications.
}

This is an open access article under the CC BY-NC-ND license. (http://creativecommons.org/licenses/by-nc-nd/4.0/)

\begin{abstract}
Modular Self-Reconfigurable Robotic (MSRR) schemes are a vital remedy for the developing demand in consumer products, automations and space exploration. The wider factor of usage and self-healing capacity are some of the influencing characteristics of robot for actual-wide application whereas segmental robotics provide an effective remedy with respect to traditional robotics. Researchers have now noted different forms of application and prototyped different systems of robotics whereas concentrating on constraints such as homogeneity, configurability, energy consumption and form factor. Diversified condition of various segmental robotic remedies projected for actual-world application and usage of various actuators and sensors interfacing methods alongside physical optimization of models present potential problems whereas visualizing and identifying the advantages and disadvantages of various approaches to remedies. This research reviews the various self-reconfigurable robotic schemes with a brief overview of history and architecture of the robotic schemes. Later in this contribution, the problems in the design of hardware, control and planning algorithms, mixed hardware and software problems and in its application problems that are underway are critically evaluated with respect to modular self-reconfigurable robotics.
\end{abstract}

Keywords - Modular Self-Reconfigurable Robotic (MSRR), Freedom Degree (FD), Shape Memory Alloys (SMAs).

\section{INTRODUCTION}

Modular Self-Reconfigurable Robotic (MSRR) Schemes focus on motion planning, design fabrication, automation control of kinematic machines with mutable morphology [1]. Aside from conventional actuations, control and sensing identified in static morphology robot, self-reconfigurable automatons can transform their shapes through the arrangements of their connections to effectively adapt to any environment, complete particular tasks and recovery from any potential damages. In recent years, segmental robotics has developed significantly with respect to the 'proof of concept' schemes with a purpose of elaborating physical simulations and implementations.

The main purpose of this contribution is to evaluate some of the modular self-reconfigurable robotics and identify the problems and opportunities, which are underway. Segmental automatons are typically incorporates with many building blocks of smaller repertoires with the same docking interface, which permits the transmission of mechanical force, electric power and communication schemes through an automation. Segment structuring blocks normally incorporate some major structural actuators and specialized units e.g. generation units, energy storage, payload, cameras, wheels, feet and grippers. MSRR can be categorized into various architectural segments by geometrical classification of units [2]. Various schemes display hybrid features, which have been discussed below.

- Lattice architecture: Lattice architecture incorporates units, which have been organized and linked to the same standard 3D patters i.e. modest hexagonal and cubic grid. Motion and control can be considered in a parallel form. Lattice architecture typically provides modest configuration, as a component shift meant to discrete categories of close geographies whereby motion can be possible and available as an open loop. Computational representation could be scaled with ease and changed into more complex systems.

- Tree/Chain architecture: Tree and chain architecture incorporate units, which are linked to tree or string architecture. Tree and chain could be folded and considered to be filled with spaces. Nonetheless, the structured are serial. The chain structures can attain any points of orientations and therefore multipurpose and challenging to analyze, represent and control.

- Mobile architecture: Mobile architecture has units, which utilize the ecosystem to maneuvers and might possibly hook more complex chains and lattices to create Smaller automatons, which execute coordinated motion to create a massive virtualized network. The controls of three different forms of segmented systems can be decentralized and redistributed among different components and might be executed in various forms of series and in a more parallel manner. Using the scheme incorporated in the modern age are incorporated of rigid elements, deformable units and complaint techniques have been explored widely. MSRR can be categorized based on the units that have been moved or reconfigured [3]. 
- Deterministic reconfigurable: This form of re-configuration depends on the units shifting or manipulated into target environments through re-configurations. The precise location of every unit is identified every moment and might be calculated or discovered during runtime. Moreover, reconfigurable moments can be assured. Feedback controls is fundamental to assure the exact manipulation; for instance, in mobile and chain architecture.

- Stochastic configuration: This type of configuration is dependent on the shifting of units with respect to statistical processes. Precise locations of each unit are noted whenever it is connected to major structures; however, it might pursue unidentified moments, which are assured statistically. Stochastic architecture is favorable at a microscale. The environment, whether fabricated or natural provide more energy for transferring the components in different forms of systems. Other types of segment robotics are present but are not self-reconfigurable and therefore do not belong to the robotic family; however, they might share the same control and design problems [4]. For instance, self-assembling schemes might incorporate multiple elements; however it cannot vibrantly reconfigure or control target shapes.

Tensegrity robot might incorporate many components that can be substituted; nonetheless, are not configured. The swarm robot incorporates many units; however, they are not typically linked to create complete physical frameworks. In addition, mechanical automatons that have tool exchangers could be viewed in form of segments; nonetheless, the measure in which they can reconfigure with ease is limited and based on the various forms of systems as evident in this research. There are three fundamental motivations for formulating MSRR:

- Versatility: MSRR are possibly more adaptive compared to the convectional systems. The capability of configuration allows groups of robots or single robots to dissemble or reassemble machines for form new types of morphologies that can suit and undertake different tables e.g. changing itself from robotic systems that are legged to those that are snake-like or robots that roll.

- Robust: Due to the fact that robots can be segmented, (in the automatons or between automatons), machines can be incorporated and replace damaged parts hence resulting to self-repair.

- Affordability: Robotic schemes that can be configured with ease can potentially lower the overall expenditure on robots through the creation of multiple copies of a single form of elements, so mass production and economies of scale are considered. Moreover, the varied range of complex machines can effectively be made from a single module segment, enhancing affordability through the aspect of generality and re-utilizing systems.

The merits evaluated in the above part have not fully been attained. The incorporated degree of freedom makes segmental automatons to be versatile in their possible capacities; however, they face performance trade-off and computational and mechanical complexities. Segmental automatons are likely to be inferior based on their performance compared to a single form of custom robot that has been tailored for a particular task. Consequent to that, the advantages of segmental automatons is apparent whenever focusing on many tasks that might typically require the collection of different coupled morphological robots and whenever the task condition cannot be forecasted beforehand the robots being deployed.

Based on the advantages discussed above, what are the major application areas for MSRR? Whereas schemes have the capacity to complete various tasks, identifying 'killer application' has been more elusive. Space exploration is one of the applications in this case. Long-term space mission necessitate self-sustainable robotic ecology, which can deal with unforeseen conditions and might necessitate self-repairability. Self-reconfiguration systems are more effective and can handle various tasks that are never identified, mostly contrasted to fixed configuration schemes. Moreover, space missions are highly constrained with respect to mass. Thus, sending robotic schemes, this can reconfigure to accomplish multiple tasks can save shipping volume and mass that sending multiple automatons, which can accomplish the same tasks [5].

This section has introduced the concept of MSRR and discussed the architectures and advantages of robotic schemes. The advantages have been drawn with respect to real-life application i.e. space exploration. The sections below focus on the various review of segmental self-reconfigurable robotic schemes and potential problems in the design of hardware, control and planning algorithms, mixed hardware and software problems and in its application problems. The remaining part of the contribution is organized as follows: Section II presents the background and history of the research. Section III evaluates the relevant literature sources of the research. Section IV presents a critical analysis of the research: MSRR and discussion of application problems. Lastly, Section V concludes the research and proposes future research directions.

\section{BACKGROUND AND HISTORY}

The history of MSRR concept is traced back from the 'prompt transformation' end effecter and automated tool 'changer' in computing numerical-based machining center dating back in the early $70 \mathrm{~s}$. In this case, special elements with the come connection mechanisms will be swapped on the end robotic arms. With reference to the foundational concept of the similar connection approach and with the application of full automations, which include cell robots structured in the late 1970s. During the early 1980s, Greg Chirikjian who structured the lattice configuration systems and the chain-based systems made significant developments. These are researchers that began with their emphasis on mechanical engineering, building and designing elements and then developing the codes that programmed them.

Wei-Min Shen and Daniela Rus developed hardware; however, it has a significant implication on the aspect of programming. They began the trends to more verifiable and provable distributed algorithm for the control of massive number of elements, incorporating dynamically discovering topological transformations and shifting habits based on 
novel topologies. Once of the most appealing hardware platforms that was developed over the past few decades has been MTRAIN (segmental transformer) series, by Murata Satoshi. This was a scheme that had hybrid chains and lattice schemes. It has the best of all of these schemes: best tasks performer of the chain schemes that is mixed with the best reconfigurable performances of the lattice scheme.

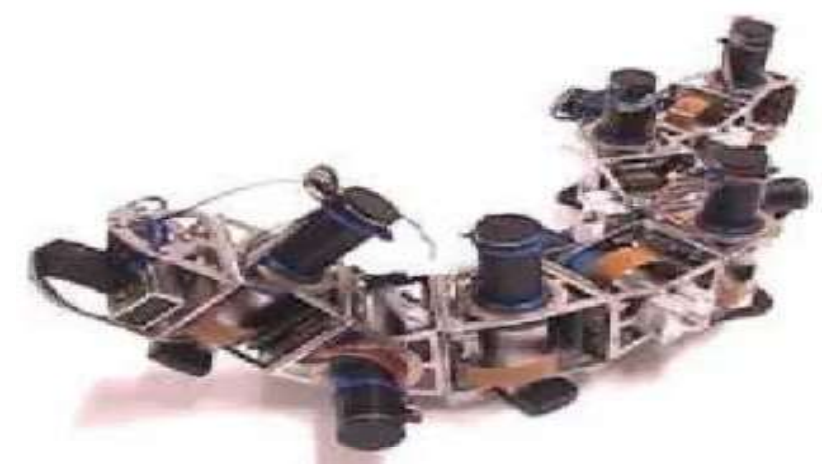

Fig 1: G2 (nine elements) connected in a snake-like configuration. Micro video camera incorporated with 9V battery and transmitter in front.

The designing concept of PolyBot is that every module is modest and that it cannot accomplish much by itself. When compared to other complex schemes, it can accomplish tasks that are more complex. Another design objective of PolyBot is that every module has to fit in the cubes of $5 \mathrm{~cm}$ on sides. Three PolyBot scheme have been established and tested. The first one was known as the $1^{\text {st }}$ generation $(\mathrm{G} 1)$ that is the modest prototype, which incorporated hobby RC servos. The constructions were constructed with the use of laser cut plastics. At least 32 elements were structured and controlled through gait-control tables with computing that is off the board. The $2^{\text {nd }}$ generation $(\mathrm{G} 2)$ functionalities incorporate more reconfigurable capacity, more strength and on-board computing (see Fig 1).

The $3^{\text {rd }}$ generation (G3) module is presently being developed (see Fig 2). G3 is more compact compared to G2 and incorporates ratchet and brake to the major actuators.

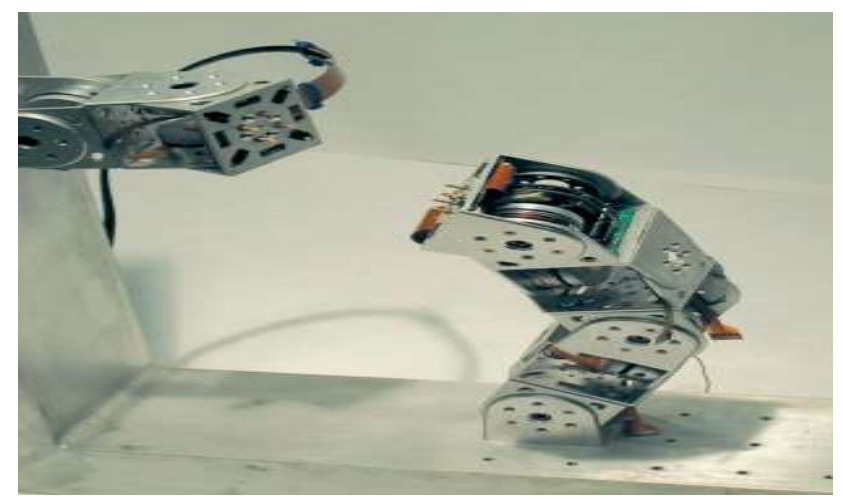

Fig 2: G3 structuring of PolyBot

PolyBot signifies MSRR with two forms of elements: Node and Segment. The segment module incorporates a single Freedom Degree (FD) and two connector ports. On the other pointer, node module is more rigid with no internalized FD and six connector ports. Schemes have concentrated on focusing on versatility problems. The future generation will focus on robustness and affordability issues.

Over the past few decades, novel ambitions in stochastic assembly have been done by Eric Klavins and Hod Lipson. Many elements have been simulated with a number of hardware module demonstration considered. The works base on the demonstration of Penrose in the early 1950s and recent chemistry works by G. Whitesides. Significant efforts have been followed to structure more elements that focus on the simplification of hardware with respect to scalability problems. Now, they have utilized wide-range simulation with less hardware module prototypes. Most of the tasks have been accomplished with ease with respect to chain reconfigurable module. This shows the versatility of the schemes. Nonetheless, other two merits (low cost and robustness) have not been pursued.

Generally, prototype scheme structured in the laboratories have been expensive and fragile. Presently, there is an advancing group of research focussing on the scheme and two critical problems in robotics. Algorithmic development has complemented hardware enhancements. Now, MSRR have been established in addition to PolyBot, and this will be evaluated further in the later section of this contribution. Section III below focusses on the literature survey of MSRR with reference to their designs and structure.

\section{LITERATURE REVIEW}

Y. Fei and C. Wang in [6] structured a 2D lattice class of MSRR known as Fracta. The robot in the lattice class incorporates the top, middle and bottom module that has permanent magnet. The middle one has electro-magnet. The process of docking starts with the incorporation of the middle segment into the empty spaces between the bottom and 
top layers of the nearest module by electromagnet activation. The operating rule was evaluated based on the application module that is based on castors on surfaces with no friction.

A. Spröwitz, R. Moeckel, M. Vespignani, S. Bonardi and A. Ijspeert in [7] structured a 3D molecule structure, every unit in the structure incorporate two different atoms, and angular bond links all of them. A linker that is an electromagnet is available on the side face of every single atom. Dual atoms, which have been connected are known as molecules and every single atom incorporates a dual Freedom Degree (FD) with a single form provided by motors at linkers of faces and another one because of motors at bonds. Molecules in general provide four FD and could be used for the formation of arbitrary structuring such as fortification.

S. Chennareddy, A. Agrawal and A. Karuppiah. in [8] prototyped the 3D unit as a cubical structure incorporating the linkers on the various faces. In the structure, every face can revolve independently alongside their axis giving 3D unit of six FD. The linkers on the various faces are linked to one 7W motor based on the worm gear mechanism that is governed by the same solenoid-based switching method for every arm. The arm is linked with the connection cuff that can move to and from along the arm axis. The connection pointer mounted of the cuff can close at a single extreme of the slide displacement to open another.

A. Kataria, H. Özbay and H. Hemam in [9] proposed I-cubes, which is another cubical structure of the robotic structure that has two dissimilar units of a cube and link. The faces of the cubes incorporate the female link with the capacity of mounting a link with a key via the lock mechanisms. Cubes at a certain timeframe can include zero to six links connected to a particular face. A link is controlled self-sufficiently with respect to multi-joints, transferred and shared between various cubes. Horizontal link beam incorporates the joints at the middle of the dual horizontal beam and can be revolved. Cubes can revolve based on the links after any successful latch and therefore giving locomotion to the cube in the scheme.

F. Lian And P. Lin In [10] established a mini-form design known as micro-unit, which is prototyped as a dual model and each module in these system include skeletons, which are square in shape with dual static female linking the ends of the rotating and diagonal male. The male part links the parts of the end of the diagonals. The first prototype structured can create the construction in 2D with dockages incorporated using tensional springs incorporated from the Shape Memory Alloys (SMAs). These constructions have torsions and a spring incorporating SMAs for generating a rotational approach. O. Ostertag and E. Ostertagová in [11] also miniaturized elements by elimination control units available in the initial prototype. This can therefore structure the second model with the capacity to create constructions in $3 \mathrm{D}$.

X. Gao, S. Jiang, L. Ren, X. Jiang, G. Chen and R. LUO in [12] evaluated vertical robot in the cubical system of 89 $\mathrm{mm}$ self-controlled unit. Each cube incorporates two similar pointed on the parallel side of the face and these pointer are the same to that of humans and the faces have magnetic sheets. Cells have the capacity to extend pointers and rotate along the axis that is perpendicular to the surface it has been mounted on. Designs facilitate the locomotion of automatons along a vertical plane and therefore stacks are just a method that has been support for motion. Pointers of the dual robot design are docked for lifting and the method of docking is based on the gender lock and the key passive linkers. Pointer extension is based on the sliding technique.

T. Yoshida et al. in [13] evaluate crystalline, which is a cuboid stricture of automatons with retraction and expansion capacity on the side face. The retraction and expansion of faces are done on the various faces simultaneously with respect to pinion and rack technique. Active connection method is available on dual neighbouring side face and passive linkers approach is available on others. Due to the fact that they systems are not structured on docks available on both the bottom and top of the faces, MSRR structures that are crystalline in nature have cases that are 2D in nature.

Y. CAO, W. GE and H. ZHANG in [14] structured Telecube elements, which is a development from the crystalline design that supports the 3D structure. Every face on the telecube module has been segmented into four quadrants and incorporate magnet poles piece in the odds and magnetic metals available in evens. They also incorporate the borders that have been chamfered for passive dockage. The components are fixed if they are closer to one another because the linker plate are SMAs springs while mirrored pictures are present in these schemes pulling magnetic poles pieces in for undocking.

J. Panasiuk and M. Soroczyńska. in [15] evaluated the Cubic structured elements, EM cubes, which also incorporated electro-magnet on the four various faces for locomotion and docking. Perpetual electromagnet include provides stronger connection and electromagnets to enhance movement. The electromagnets have been placed to formulate attractive and repulsive force simultaneously to produce fixed forces at both ends of the cube to enhance movement.

H. Kurokawa, K. Tomita, A. Kamimura, S. Kokaji, T. Hasuo and S. Murata in [16] structured the M-block cubical MSRR that has been prototyped as dual version: 3D M-block and M-block. 3D M-block and M-block have inertial actuators in the middle of the block for governed torque at the centre of mass modules and thus rotating the $\mathrm{m}$ blocks MSRR in all directions.

S. Motavalli and B. Bahr in [17] evaluated the M-block cell with the capacity of motion for coordinate location and docking. The edges and faces of the models have been embedded with permanent magnet. Rapidly decelerating and accelerating internal rotation approaches source edge electro-magnet and locomotion utilizing pivot actions. Face electro-magnet are capable of supporting the alignments available in the components based on various aspects of movements. $\mathrm{M}$ blocks provide proper actuations in a single locus and three-dimensional $\mathrm{m}$ block can potentially stimulate it in 6 different directions using a change in inertial actuator in 3-orthogonal axes for three-dimensional locomotion.

O. Kosak, C. Wanninger, A. Hoffmann, H. Ponsar and W. Reif in [18] presented the mini factor MSRR, MICHE, which has been structured for creating lattice structure in $3 \mathrm{D}$ with respect to the ecosystem. Different faces of the cubic- 
type miche are structured using a magnet that can be switched and the remaining portion of faces are incorporated in the plates made of steel. Electromagnets have been placed away from the geometrical segment of the plates for removing the forces repelling the electromagnet of two-robotic structures during a docking process. Magnet switching is controlled using inner microcontrollers linked using IR transceiver and thus giving the ability that retains the structures to miche MSRR. These are classified for its dependency on the environment for locomotion and aggregation.

Section III has evaluated the constructions and design of MSRR. Section IV provides a critical analysis of various self-reconfigurable scheme and potential problems of robotics.

\section{CRTICAL ANALYSIS}

\section{Self-Reconfigurable Schemes}

UBot

UBot MSRR scheme incorporates cubic structured cell with the capacity of revolving in discrete phases alongside a lengthier diagonal, which is the same at molecules. The internalized faces have been chamfered for facilitating rotations. UBot robotic cell is classified into passive and active elements with more active module that provide active linkage interface and passive module with four passive interlinkage interfaces. Passive and active elements have uniform outer rotating and constructions mechanism. Hooks available on active interlinking interfaces allow stable docking with passive connections. Passive and active elements are latching using shifting and hook mechanism controlled by the sensor position for creating chain and lattice constructions in 3D structuring of UBot, which is a hybrid class of automatons.

\section{RoomBots}

Roombots are another hybrid architecture that have been structured from lattice and chained constructions in 3D. Each room-bot include dual cells in a spherical structure that are linked. Every cell is an interlinkage of two half-circles on every other alongside the faces as indicated in Fig 3. Motion is based on three gear motors: One is available at every cell for rotating other half circles and the other one is at the connections between the cells. Every toombot robot has ten vibrant interlinkages from the elements that are closer to one active connection from nearly half a sphere and eight different connectors that are passive. The interlinking method between different room-bots are assigned using different latches to sustain the nearest elements at the various holes available on the surface,

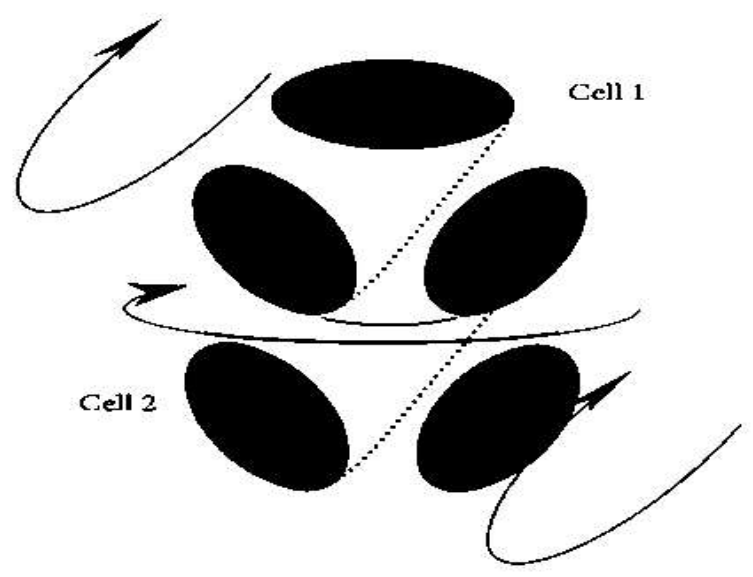

Fig 3: RoomBot MSRR diagram

Soldercubes

Soldercubes represent a hybrid segment of MSRR with the shape of a single soldercube that is the same to the cell in a dual cell roombot structure. Six different interlining faces, that have no gender for the cells, facilitate the process of docking in between the coordinated motions and modular motions. The connecting faces are structured with respect to boards using soldering. Linkers' contacts can melt with ease upon the transmission of current at low temperatures, therefore establishing bonds between elements for creating constructions alongside electrical and mechanical connections. Bonds can be broken based on the same mechanisms of contact melt. Soldercubes elements have incorporated mechanisms meant for rotations of the same connector faces providing one FD to the components; however, facilitates different FDs after it docks with the same module.

\section{PolyBot (G3)}

According to Fig 4, Poly-bot was created at the research institute of Palo Alto. The robot a chained configurable system whereby every module was structured in a cubical manner, was about $49 \mathrm{~mm}$ on its sides, and had one rotational FD. It is known as a segment of Poly-Bot that has proven various modes of motion incorporating: snake-like, walking, and 14legged slinky; concertina as gopher holes, inch worm gait, rectilinear undulations and side-winding gait, rolling-like treads of about $1.61 \mathrm{~m}$ per second; riding tricycles and climbing tricycles. Elements incorporate the brush flat motors 
that have harmonic drivers, pressure torque sensors, whisker-touched sensors, and the infrared nearness sensors. These use hermaphroditic connector that has SMAs actuated latch.
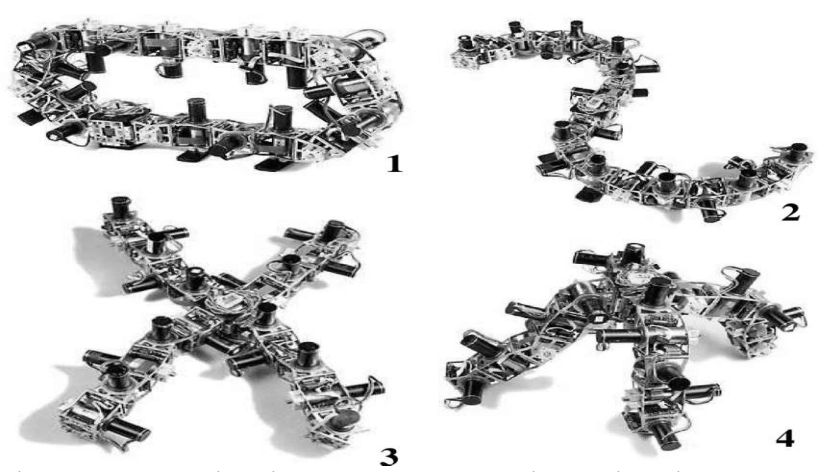

Fig 4: PolyBot, as seen in Fig 4, was structured at Palo Alto Center of Research

\section{Programmable Parts}

Programmable parts (2005), in Fig 5, have been structured to explore the amounts of chemical reactions that can be programmed. Programmable segments are stirred in a random manner on air hockey tables through actuated air jet. Whenever they stick and collide, they can potentially decide and communicate whether to remain stuck or when they have to delink. Contained guidelines for interaction could be optimized and effectively devised to govern roots to transform into different shapes. Systems that have been structured by these contained guidelines could be modelled based on chemical master equations and the evaluation of its habits following the standard ideologies in a nonequilibrium statistical dynamics. Resultant theory being developed is applicable to microscales assembly and molecular self-assembly Ultimate goals are to comprehend the manner in which program stochastic assemble can be drawn to scale.

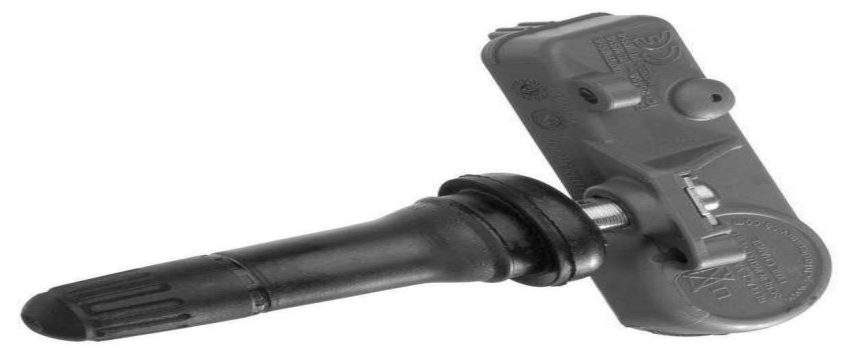

Fig 5: Representation of Programmable parts (2005)

\section{Molecubes}

Molecubes, 2005, as seen in Fig 6, indicate a scheme that was structured to demonstrate kinematic custom reproduction. Each type of module is approximately $0.61 \mathrm{~kg}$ cube, which is about $100 \mathrm{~mm}$ in overall length and one rotating FD. Rotational axis is accurately aligned to the diagonal of the cubes. Manual reproductions of 4-module robots are evaluated and their theoretical availability of arbitrarily-centered replication machines have been represented using a mathematical approach. Replication morphologies and controller have been presented in a spontaneous manner in simulations of primordial soup of various 2D molecubes automata.

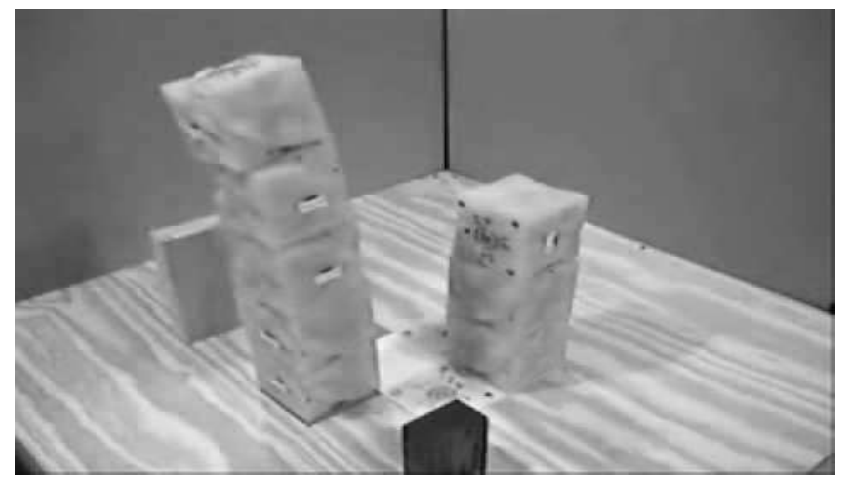

Fig 6: Representation of Molecubes, 2005

SuperBot 
SuperBot, 2006, in Fig 7, have been developed as a reconfigurable system that can be deployed for practical applications. The components incorporate lattice system and hybrid chain. The component have three different FDs (rolls, yaws and pitches) and can potentially connected to one another using six different dock connectors that are identical. For high-level control and communication, elements utilize applicable operational systems and hormonecentred control structured for the controlling purpose scalable and distributed protocol, which does not necessitate elements to have unique identities.

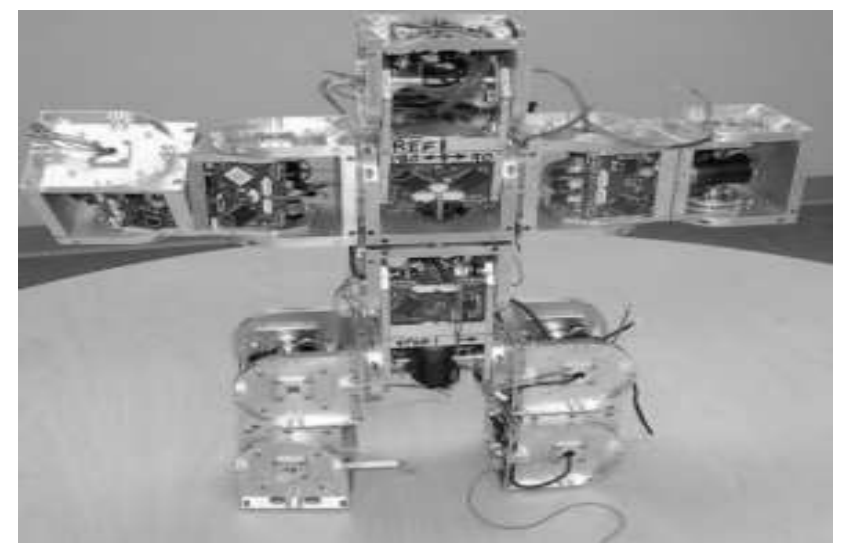

Fig 7: Representation of SuperBot, 2006

\section{Miche}

The Miche scheme, as seen in Fig 8, was built as a module lattice scheme with the capacity of arbitrary shape creation. The scheme attains the assembly through the process of disassembly and potentially establishes robust operations after conducting different laboratory experiments. Each type of module is considered an automatic robotic cube with the capacity to interlink and communicate with its closer neighbors. Connecting techniques are given by switchable magnet. Elements utilize face-to-face communication, which is applied together with infrared systems to possibly identify the present of closer neighbors. When gathered into the essential constructions, the components create schemes, which can be sculpted based on the application of computer interfaces and distributed procedures. Groups of elements collectively decide who is available on final shape using an algorithm, which can minimize data storage and transmission. Finally, elements not in constructions fall off or let go under the control of external forces, i.e. gravity. Algorithms governing these procedures are distributed and considered effective in their spaces and communication usage.

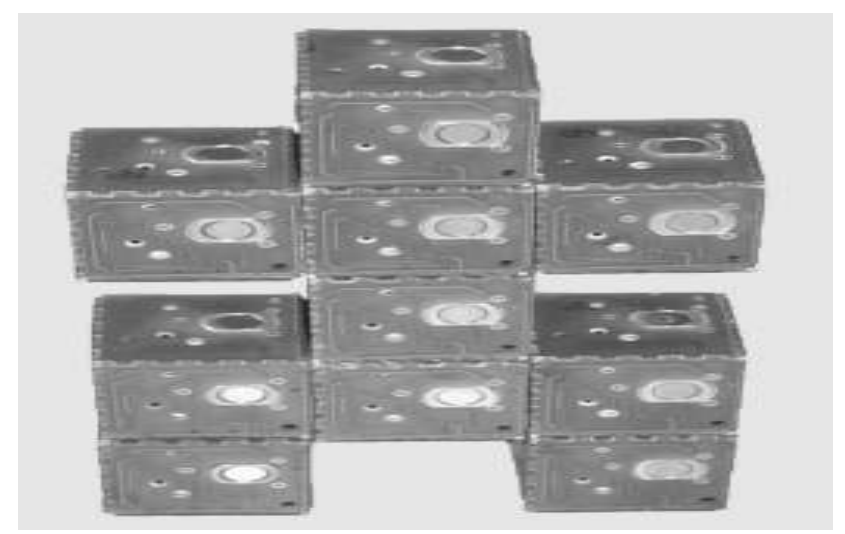

Fig 8: Representation of the Miche schemes

Discussion of Potential Problems

Referenced from early demonstrations of MSRR, performances have considerably increased with reference to sizes and robustness. Dimension to which the promise of self-reconfigurable robotic scheme is fulfilled is based on the module scheme. Presently, schemes with approximately 49 units have been shown with different numbers staged for many years. Planned and controlled algorithm has witnessed significant advancement to potentially handle hundreds of units. Nonetheless, different steps, that are fundamental for the systems' need to fulfill their demand of affordable, adaptable and robust factors have been discussed above. These steps can be categorized into problems in the design of hardware, control and planning algorithms, mixed hardware and software problems and in its application problems.

Problems in the Design of Hardware

Performance of the self-reconfigurable automatons is significantly based on electronic and mechanical design. Presently, different designs have undergone significant assessment and development. Each of the designs have concentrated on the various factors e.g. form factor flexibility, applying FD, high torque to the ratios of weights, 
docking ease, undockage and power control. However, optimum and general- purpose designing of modules has not been proposed yet. Critical limiting factors, which control the issue include:

- Strength limits, field robustness (i.e. both electrical and mechanical) of docking/bonding interfaces in the elements and precision.

- Limitations of motor power, precision of motion and energetic efficacy of components (particular torque and particular energy input)

- Demerits on dexterities of individual components that is capable of limiting flexibility of automatons in general.

\section{Control and Planning Algorithms}

Even though algorithms have been structured to deal with various units under certain conditions, issues of scalability remain on the high-level planning and low-level control to mitigate actual constraints e.g.:

- An algorithm for more parallel motions for large-scale aspects for motion considering various obstacles.

- An algorithm for optimum (time and energy) reconfigurable planning with or without an obstacle

- An algorithm for robustly dealing various failure nodes from misalignment and mortal units, which are irresponsive or do not release to the units that can behave in an erratic manner.

- An algorithm, which can potentially determine optimum configurations fulfilling the demands of certain tasks with reference to the ecosystem; and

- Scalable and effective communication linking various system units

\section{Mixed Hardware and Software Problems}

MSRR have considerably coupled software and hardware compared to other schemes. In that case, there are various problems for which remedies can fall on for either software or hardware categorization. These problems incorporate:

- The obligation of the sensor for reconfigurable robots: For different types of applications, the components of reconfigurable robots need to be cognizant with respect to their respective ecosystems based on widerange sensors. Because of the distributed condition of the module network, sensor data is present in a distributed manner and therefore this data has to be linked for more automated processes of making decisions or communicated to rural controllers' hosts.

\section{Application Problems}

Other than technical issues, there are other non-technical issues considered as well. Even though the merits of MSRR have significantly been structured, it is nearly difficult to identify certain types of domains of application whereby they merits can be visualized. It is considered that identifying applications, which clearly drive the requirement for these schemes, is the potential issues. Segmental automatons can, in future, be utilized in various practical applications whereas adaptive and unsupervised self-organized is fundamental. Various key technical problems are evident, nonetheless. In this segment, we will evaluate the various critical problems that, if mitigated, will allow the future generation of MSRR to have wide-range capacities.

\section{Larger systems}

Various systems of more segmented robots have been less; for instance, the elements that exist in living cells (that most of the researchers considered as the best MSRR example). The demonstrations of the schemes with about a thousand personal units could recommend that these segmented robots are old. Manual demonstration of these schemes will necessitate brainstorming key hardware problems, e.g. binding mechanisms, dynamics, vibrations and power distributions. It will also necessitate novel distribution algorithms, which account for failures, errors, noise, transforming connector topology.

\section{Self-repairing schemes}

Other than reconfiguring itself into novel shapes, schemes incorporated of segmental robotic schemes may be able to recover from critical damages e.g. the one that might result from externalized failure or internalized collision. Demonstration of self-healing constructions comprising of multiple communicating, distributed segments could require a critical algorithm that can estimate and sense intercontinental conditions, including robust algorithms and hardware for reconfigurable, which operate based on initial conditions. Concrete examples would be incorporating scheme blown up (segmented into various pieces) and therefore recover or self-assemble from the failures of a particular degree of faulty unit.

\section{Self-sustaining schemes}

Over the past few years, NASA pushed up the concept known as RoboSphere, which focusses on building selfsustaining robotic environment, isolated for a longer time and requires sustainable operations and accomplishment of unanticipated tasks without the input of humans. The present condition of the urbane segmented robots is not in anywhere closer to this objective, and, in that case, demonstrations of schemes operate for a single year. Novel methods in energy control and energy harvesting could be fundamental, including the ability to handle potential challenges that could occur over a long period. 


\section{Self-extension and self-replication}

Whereas modest robotic replication has been represented using less high-level modules, potential issues have been seen for replication practices based on different low-level components, and out of raw materials and elements that are elementary. These systems would potentially establish more active components and more reflexive constructions leading to self-replication and self-advancing systems for eco-friendly sources. Seed category demonstrations of segmental robotic schemes, which establish copies of themselves out of the raw materials, would necessitate advancing past their complexity levels known as equivalence of system-sound barrier.

\section{CONCLUSION AND FUTURE DIRECTIONS}

Segmental robotic schemes are examples of various types of self-planning systems from expert nanotechnologists and biologists. However, there are differences where various present systems have overcome entropies through brute forces or significant amounts of energy. Segmental schemes, contrary to that, focus on random diffusive procedures in critical forms. Moreover, there are completely robust to intrinsic noises identified at nanoscales. In case segmented robots need miniaturization to either microscales or nanoscales, or if the ideology discovered in the ecosystem is linked to nanotechnology, conditions of stochastic of nanoscale systems need to be discussed. System demonstration where stochastic fluctuation is considered as a predominant element, it would then signify critical advancement. This contribution has discussed various issues e.g. problems in the design of hardware, control and planning algorithms, mixed hardware and software problems and in its application problems. More issues will be discussed in the magazine of robotics and automations, dedicated to self-repairing and replication, by IEEE

MSRR have considered following critical technological advancements in the segment of robotics. Their aim of high value, high robustness and higher adaptability might lead to more radical change in automations. Presently, many researches have discussed many issues. Whereas some advancement has been initiated, it is evident that problems are still evident. Through the illustration of some of the prevailing problems as grand issues, this contribution has shown a critical direction for the future of robotics. However, future research should focus more on problems of versatility, affordability and robustness.

\section{References}

[1]. G. Chirikjian, A. Pamecha and I. Ebert-Uphoff, "Evaluating efficiency of self-reconfiguration in a class of modular robots", Journal of Robotic Systems, vol. 13, no. 5, pp. 317-338, 1996. Available: 10.1002/(sici)1097-4563(199605)13:5<317::aid-rob5>3.0.co;2-t.

[2]. D. Bie, Y. Wang, Y. Zhang, C. Liu, J. zhao and Y. Zhu, "Parametric L-systems-based modeling self-reconfiguration of modular robots in obstacle environments", International Journal of Advanced Robotic Systems, vol. 15, no. 1, p. 172988141875447, 2018. Available: $10.1177 / 1729881418754477$

[3]. D. Bie, Y. Zhu, X. Wang, Y. Zhang and J. Zhao, "L-systems driven self-reconfiguration of modular robots", International Journal of Advanced Robotic Systems, vol. 13, no. 5, p. 172988141666934,2016 . Available: 10.1177/1729881416669349.

[4]. Y. Fei, Y. Zhu and P. Xia, "Analysis on Self-Morphing Process of Self-Reconfigurable Modular Robot", International Journal of Advanced Robotic Systems, vol. 6, no. 3, p. 23, 2009. Available: 10.5772/7232

[5]. T. Larkworthy and S. Ramamoorthy, "A characterization of the reconfiguration space of self-reconfiguring robotic systems", Robotica, vol. 29, no. 1, pp. 73-85, 2011. Available: $10.1017 / \mathrm{s} 0263574710000718$.

[6]. Y. Fei and C. Wang, "Self-Repairing Algorithm of Lattice-Type Self-Reconfigurable Modular Robots", Journal of Intelligent \& Robotic Systems, vol. 75, no. 2, pp. 193-203, 2013. Available: 10.1007/s10846-013-9885-8.

[7]. A. Spröwitz, R. Moeckel, M. Vespignani, S. Bonardi and A. Ijspeert, "Roombots: A hardware perspective on 3D self-reconfiguration and locomotion with a homogeneous modular robot", Robotics and Autonomous Systems, vol. 62, no. 7, pp. 1016-1033, 2014. Available: 10.1016/j.robot.2013.08.011.

[8]. S. Chennareddy, A. Agrawal and A. Karuppiah, "Modular Self-Reconfigurable Robotic Systems: A Survey on Hardware Architectures", Journal of Robotics, vol. 2017, pp. 1-19, 2017. Available: 10.1155/2017/5013532.

[9]. A. Kataria, H. Özbay and H. Hemami, "Controller design for natural and robotic systems with transmission delays", Journal of Robotic Systems, vol. 19, no. 5, pp. 231-244, 2002. Available: 10.1002/rob.10037.

[10]. F. LIAN and P. LIN, "MODULE DESIGN WITH COMMUNICATION AND RECONFIGURATION FOR SNAKE-TYPE MODULAR ROBOTIC SYSTEMS", International Journal of Information Acquisition, vol. 07, no. 03, pp. 205-223, 2010. Available: $10.1142 / \mathrm{s} 0219878910002166$.

[11]. O. Ostertag and E. Ostertagová, "Shape Memory Alloy Actuator (SMA)", Applied Mechanics and Materials, vol. 816, pp. 9-15, 2015. Available: 10.4028/www.scientific.net/amm.816.9

[12]. X. GAO, S. JIANG, L. REN, X. JIANG, G. CHEN and R. LUO, "Experimental Research on Self-adaptability of Tri-axis Differential In-pipe Robot Drive Unit", ROBOT, vol. 32, no. 3, pp. 419-424, 2010. Available: 10.3724/sp.j.1218.2010.00419.

[13]. T. Yoshida et al., "Benefits of side-by-side deployment of 6-mm covered self-expandable metal stents for hilar malignant biliary obstructions", Journal of Hepato-Biliary-Pancreatic Sciences, vol. 23, no. 9, pp. 548-555, 2016. Available: 10.1002/jhbp.372.

[14]. Y. CAO, W. GE and H. ZHANG, "Structure Design and Simulation Analysis of an Innovative Modular Self-Reconfigurable Robot-360bot", Robot, vol. 35, no. 5, p. 568, 2013. Available: 10.3724/sp.j.1218.2013.00568.

[15]. J. Panasiuk and M. Soroczyńska, "Design of walking robot model moving on vertical areas", Mechanik, vol. 90, no. 7, pp. 637-639, 2017. Available: 10.17814/mechanik.2017.7.97.

[16]. H. Kurokawa, K. Tomita, A. Kamimura, S. Kokaji, T. Hasuo and S. Murata, "Distributed Self-Reconfiguration of M-TRAN III Modular Robotic System", The International Journal of Robotics Research, vol. 27, no. 3-4, pp. 373-386, 2008. Available: $10.1177 / 0278364907085560$.

[17]. S. Motavalli and B. Bahr, "Automated tool monitoring system using vision system for a robotic cell", Robotics and Computer-Integrated Manufacturing, vol. 10, no. 4, pp. 311-319, 1993. Available: 10.1016/0736-5845(93)90044-k.

[18]. O. Kosak, C. Wanninger, A. Hoffmann, H. Ponsar and W. Reif, "Multipotent Systems: Combining Planning, Self-Organization, and Reconfiguration in Modular Robot Ensembles", Sensors, vol. 19, no. 1, p. 17, 2018. Available: 10.3390/s19010017. 